\title{
A retrospective observational study of clinicopathological features of KRAS, NRAS, BRAF and PIK3CA mutations in Japanese patients with metastatic colorectal cancer
}

Akihito Kawazoe', Kohei Shitara ${ }^{1,2}$, Shota Fukuoka', Yasutoshi Kuboki', Hideaki Bando', Wataru Okamoto 1,2, Takashi Kojima', Nozomu Fuse', Takeharu Yamanaka ${ }^{2,3}$, Toshihiko Doi ${ }^{1,2}$, Atsushi Ohtsu ${ }^{1,2}$ and Takayuki Yoshino ${ }^{1,2^{*}}$

\begin{abstract}
Background: The mutation in KRAS exon 2 is a validated biomarker of resistance to anti-epidermal growth factor receptor (EGFR) therapy in metastatic colorectal cancer (mCRC). Several reports have confirmed associations of other RAS mutations with resistance to anti-EGFR therapy. However, the impact of BRAF and PIK3CA mutations on the efficacy of anti-EGFR therapy remains controversial. Little is known about the frequencies and clinicopathological features of these mutations, as well as the therapeutic effects of anti-EGFR therapy in mCRC patients with these mutations, especially in the Asian population.

Methods: In this retrospective observational study, frequencies and clinicopathological features of KRAS, NRAS, BRAF and PIK3CA mutations were evaluated in patients with mCRC. Among patients treated with anti-EGFR therapy, objective response, progression-free survival (PFS), and overall survival (OS) were evaluated according to gene status. Results: Among 264 patients, mutations in KRAS exon 2, KRAS exons 3 or 4, NRAS, BRAF and PIK3CA were detected in $34.1 \%, 3.8 \%, 4.2 \%, 5.4 \%$ and $6.4 \%$, respectively. Thus, a total of $12.1 \%$ of patients without KRAS exon 2 mutations had other RAS mutations. Primary rectal tumors tended to be more frequently observed in RAS mutant tumors. BRAF mutations were more frequently observed with right-sided colon, poorly differentiated or mucinous adenocarcinoma, and peritoneal metastasis. Among the 66 patients with KRAS exon 2 wild-type tumors treated with anti-EGFR agents, PFS (5.8 vs. 2.2 months) and OS (17.7 vs. 5.2 months) were significantly better in patients with all wild-type tumors $(n=56)$ than in those with any of the mutations $(n=10)$. The response rate also tended to be better with all wild-type tumors (26.8 vs. 0\%).

Conclusion: Other RAS and BRAF mutations were observed in KRAS exon 2 wild-type tumors, which were associated with some clinicopathological features and resistance to anti-EGFR therapy in our patient cohort.
\end{abstract}

Keywords: Colorectal cancer, KRAS, NRAS, BRAF, PIK3CA, Epidermal growth factor

\footnotetext{
* Correspondence: tyoshino@east.ncc.go.jp

'Department of Gastroenterology and Gastrointestinal Oncology, National Cancer Center Hospital East, 6-5-1 Kashiwanoha, Kashiwa, Chiba 277-8577, Japan

${ }^{2}$ Exploratory Oncology Research \& Clinical Trial Center, National Cancer

Center, Chiba, Japan

Full list of author information is available at the end of the article
}

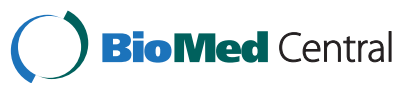

(c) 2015 Kawazoe et al.; licensee BioMed Central. This is an Open Access article distributed under the terms of the Creative Commons Attribution License (http://creativecommons.org/licenses/by/4.0), which permits unrestricted use, distribution, and reproduction in any medium, provided the original work is properly credited. The Creative Commons Public Domain Dedication waiver (http://creativecommons.org/publicdomain/zero/1.0/) applies to the data made available in this article, unless otherwise stated. 


\section{Background}

Colorectal cancer was the third most common cancer in men $(746,000$ cases, $10.0 \%$ of the total) and the second in women $(614,000$ cases, $9.2 \%$ of the total) worldwide in 2012 [1]. Mutations in KRAS exon 2 occur in $~ 35 \%$ of all metastatic colorectal cancers (mCRCs) $[2,3]$, and constitutively activate the mitogen-activated protein kinase (MAPK) pathway $[4,5]$. These mutations are validated biomarkers for resistance to anti-epidermal growth factor receptor (EGFR) therapy in patients with mCRC [6-11]. Although conventional KRAS tests are useful to exclude patients without benefit from anti-EGFR therapy, response rates and disease control rates to anti-EGFR antibody monotherapy among patients with KRAS exon 2 wild-type tumors are only $13-17 \%$ and $51 \%$, respectively $[6,7]$. Therefore, more accurate patient selection requires identification of other predictive factors to improve the riskbenefit profile of anti-EGFR therapy.

Until recently, there have been no validated biomarkers other than KRAS exon 2 mutations. Recently, several reports have shown that other KRAS (exons 3 or 4) and NRAS mutations (exons 2-4) occur in $\sim 20 \%$ of mCRC patients with $K R A S$ exon 2 wild-type tumors, which are associated with resistance to anti-EGFR therapy for $\mathrm{mCRC}$ [12-18].

$B R A F$ mutations were detected in $5-10 \%$ of patients with $\mathrm{mCRC}$ with $\mathrm{V} 600 \mathrm{E}$ as a hot spot. BRAF is a downstream molecule of $K R A S$ and the clinical data suggest that BRAF V600E mutations are associated with poor prognosis in patients with mCRC [11,12,19-24]. However, the relationship between $B R A F$ mutations and the efficacy of anti-EGFR therapy remains controversial [19-22]. Besides the KRAS-BRAF pathway, the other major downstream signaling pathway activated by EGFR is the PI3K-AKT signaling pathway. PIK3CA mutations, most of which were in exons 9 and 20, were detected in $10-15 \%$ of patients with mCRC. According to a European Consortium report [19], PIK3CA mutations in exon 20 but not in exon 9 were associated with resistance to antiEGFR therapy for mCRC. However, in other studies, no clear correlation between PIK3CA mutations and the efficacy of anti-EGFR therapy has been observed [21,22]. Meanwhile, targeting agents for these mutations are under development.

We previously reported that a multi-gene cancer panel with Luminex technology (GENOSEARCH Mu-PACK, MBL, Japan) is useful for detection of 36 mutations in KRAS exons 3 or 4, NRAS, BRAF and PIK3CA in a single reaction using 50-ng template DNA from formalin-fixed, paraffin-embedded (FFPE) specimens [25]. Importantly, the analysis of 82 samples was fully concordant with conventional direct sequencing. However, information about the frequencies and clinicopathological features of these mutations in clinical practice, including the relationship between mutation status and the efficacy of anti-EGFR therapy, especially among Asian populations, is still limited.

In the present study, we evaluated the frequencies andclinicopathological features of KRAS, NRAS, BRAF and PIK3CA mutations in Japanese mCRC patients, and assessed their corresponding effects on the efficacy of anti-EGFR therapy.

\section{Methods}

\section{Patients}

We have conducted a retrospective observational study in our institution to evaluate the frequencies and clinicopathological features of KRAS, NRAS, BRAF and PIK3CA mutations in Japanese $\mathrm{mCRC}$ patients. Principal inclusion criteria were as follows: histologically confirmed adenocarcinoma of the colon or rectum; and presence of unresectable metastatic disease.

Between January 2013 and June 2014, we analyzed 264 patients with $\mathrm{mCRC}$ who met the inclusion criteria. The study was conducted with the approval of the National Cancer Center Institutional Review Board. Written informed consent was obtained from as many patients as possible. For the deceased patients and their relatives, we also disclosed the study design at the website of National Cancer Center and gave them the opportunity to express their wills in accordance with the Epidemiological Study Guideline of Ministry of Health, Labour and Welfare in Japan.

\section{Molecular profiling and data analysis}

Genomic DNA was extracted from FFPE cancer specimens (239 primary tumors and 25 metastases). A total of 36 mutations were analyzed using Luminex (xMAP) technology (GENOSEARCH Mu-PACK, MBL), including: KRAS codon 61 (Q61K, Q61E, Q61L, Q61P, Q61R and Q61H); KRAS codon 146 (A146T, A146S, A146P, A146E, A146V and A146G); NRAS codon 12 (G12S, G12C, G12R, G12D, G12V and G12A), codon 13 (G13S, G13C, G13R, G13D, G13V and G13A); codon 61 (Q61K, Q61E, Q61L, Q61P, Q61R and Q61H); BRAF codon 600 (V600E); PIK3CA exon 9 codon 542 (E542K); codon 545 (E545K); codon 546 (E546K); and exon 20 codon 1047 (H1047R, H1047L). The lower limit of the percentage of mutant allele in the tumor samples accepted by the study was 5\%. Initially, 50-ng samples of template DNA were collected from FFPE tissue samples and were amplified using polymerase chain reactions (PCRs) with a biotin-labeled primer. Subsequently, PCR products and fluorescent Luminex beads were bound to oligonucleotide probes that were complementary to wild-type and mutant genes, and were hybridized and labeled with streptavidin-phycoerythrin. Subsequently, the products were processed according to Luminex assays and data were analyzed using UniMAG software (MBL). The 
procedure took $\sim 4.5 \mathrm{~h}$. The status of KRAS exon 2 (codons 12 and 13) was evaluated by amplification using a refractory mutation system-Scorpion assay with $1 \%$ sensitivity in a central vendor laboratory.

Patient characteristics, including age, sex, site of primary lesion, histology, site of metastases, and treatment results, were collected from medical records. Sites of primary lesions were divided into right colon, left colon, and rectum. Right-sided tumors were defined as those arising anywhere from the cecum to the transverse colon, and left-sided tumors as those arising anywhere from the splenic flexure to the rectosigmoid junction. The efficacy of anti-EGFR therapy was evaluated according to gene status in patients who met the following inclusion criteria: Eastern Cooperative Oncology Group performance status (ECOG PS) score $\leq 2$, $K R A S$ exon 2 wild type, at least one prior chemotherapy regimen, treatment with anti-EGFR either as monotherapy or in combination with irinotecan or FOLFIRI (5-FU, Lleucovorin and irinotecan), baseline computed tomography (CT) performed within 28 days of anti-EGFR therapy, initial evaluation of treatment effect via CT scan within 3 months of initial anti-EGFR therapy and adequate hematological, hepatic and renal function.

\section{Statistical methods}

Gene mutation frequencies and associations of $R A S$ or $B R A F$ mutations with clinicopathological features were estimated in $\mathrm{mCRC}$ patients.

Response rate (RR) and disease control rate (DCR; including complete or partial response and stable disease) were evaluated for anti-EGFR therapy according to the Response Evaluation Criteria in Solid Tumors (RECIST; version 1.1). Progression-free survival (PFS) was defined as the time from initial administration of anti-EGFR regimens until the first objective evidence of disease progression or death from any cause. Overall survival (OS) was defined as the time from initial administration of anti-EGFR regimens until death from any cause. For PFS or OS, patients were censored at the time of their last follow-up if they were free of disease progression or alive, respectively. PFS and OS rates were estimated using the Kaplan-Meier method, and differences among the groups according to KRAS, NRAS, $B R A F$ and PIK3CA gene status were identified by univariate and multivariate analyses using Cox proportional hazards models and presented as hazard ratios (HRs) with 95\% confidence intervals (CIs). Confounders in univariate and multivariate analyses included ECOG PS (0 vs. 1 and 2 ), numbers of metastatic sites ( $1 \mathrm{vs} . \geq 2$ ), treatment line of anti-EGFR regimens (2nd vs. 3rd) and types of anti-EGFR regimens (monotherapy vs. combination therapy).

The $\chi^{2}$ test, Fisher's exact test, Mann-Whitney $U$ test, or Kruskal-Wallis test was used to compare patient characteristics and treatment response, as appropriate. Statistical analyses were performed using IBM SPSS Statistics version 21 (IBM Corporation, Armonk, NY, USA). All tests were two-sided, and differences were considered significant when $P$ was $<0.05$.

\section{Results}

Frequencies of KRAS, NRAS, BRAF and PIK3CA mutations in $\mathrm{MCRC}$ patients

Patient characteristics and frequencies of gene mutations in 264 patients are shown in Tables 1 and 2, respectively. One hundred and thirty-three patients (50.1\%) had tumors with no mutation (all wild type). Mutations in KRAS codons 12 or 13, KRAS codons 61 or 146 and NRAS codons 12,13 , or 61 were detected in $90(34.1 \%), 10(3.8 \%)$ and 11 (4.2\%) patients, respectively. Fourteen (5.4\%) patients had BRAF codon 600 mutations, and 17 (6.4\%) had PIK3CA mutations (13 in exon 9 and 4 in exon 20). The genotypes

Table 1 Patient characteristics and clinicopathological features according to RAS mutations

\begin{tabular}{|c|c|c|c|c|c|c|}
\hline Characteristics & & $\begin{array}{l}\text { All patients } \\
(n=264, \%)\end{array}$ & $\begin{array}{l}\text { All RAS WT } \\
(n=153, \%)\end{array}$ & $\begin{array}{l}\text { KRAS exon2 MT } \\
(n=90, \%)\end{array}$ & $\begin{array}{l}\text { Any other RAS } \mathrm{MT}^{\mathrm{a}} \\
(n=21, \%)\end{array}$ & $P$ value \\
\hline Age & Median (range) & $64(32-86)$ & $64(32-82)$ & $64(38-82)$ & $68(48-86)$ & $0.32^{*}$ \\
\hline Gender & Male & $166(62.9)$ & $94(61.4)$ & $56(62.2)$ & $16(76.2)$ & $0.41^{* *}$ \\
\hline \multirow[t]{3}{*}{ Primary lesion } & Right-sided colon & $53(20.1)$ & $29(19.0)$ & $21(23.3)$ & $3(14.3)$ & $0.08^{* *}$ \\
\hline & Left-sided colon & $70(26.5)$ & $50(32.7)$ & $15(16.7)$ & $5(23.8)$ & \\
\hline & Rectum & $141(53.4)$ & $74(48.3)$ & $54(60.0)$ & $13(61.9)$ & \\
\hline \multirow[t]{2}{*}{ Histology } & Well, mod & $240(90.9)$ & $133(86.9)$ & $88(97.8)$ & $19(90.5)$ & $0.17^{* *}$ \\
\hline & Por, muc & $24(9.1)$ & $20(13.1)$ & $2(2.2)$ & $2(9.5)$ & \\
\hline \multirow[t]{4}{*}{ Site of metastasis } & Liver & $137(51.2)$ & $73(47.7)$ & $49(54.4)$ & $15(71.4)$ & $0.10^{* *}$ \\
\hline & Lung & $100(37.9)$ & $55(35.9)$ & $40(44.4)$ & $5(23.8)$ & $0.16^{* *}$ \\
\hline & Lymph node & $150(56.8)$ & $87(56.9)$ & $49(54.4)$ & $14(66.7)$ & $0.59^{* *}$ \\
\hline & Peritoneum & $52(19.7)$ & $33(21.6)$ & $15(16.7)$ & $4(19.0)$ & $0.64^{* *}$ \\
\hline
\end{tabular}

*Kruskal-Wallis test; ${ }^{* *} x^{2}$ or Fisher exact test. ${ }^{a}$ any mutations in KRAS codons 61 or 146 or NRAS. mod: moderately differentiated; MT: mutation type; muc: mucinous carcinoma; por: poorly differentiated adenocarcinoma; well: well-differentiated adenocarcinoma; WT: wild type. 
Table 2 Mutation rates of each gene in 264 mCRC patients

\begin{tabular}{llll}
\hline Gene & Wild type & Mutation type & $\begin{array}{l}\text { Mutation } \\
\text { rate (\%) }\end{array}$ \\
\hline KRAS codon 12,13 & 174 & 90 & 34.1 \\
KRAS codon 61, 146 & 254 & 10 & 3.8 \\
NRAS codon 12, 13, 61 & 253 & 11 & 4.2 \\
BRAF codon 600 & 250 & 14 & 5.4 \\
PIK3CA exon 9, 20 & 247 & 17 & 6.4 \\
\hline
\end{tabular}

of all samples using HGVS nomenclature are shown in Additional file 1. All mutations were mutually exclusive except for those in PIK3CA, and 21 (12.1\%) patients without $K R A S$ mutations in exon 2 had other $R A S$ mutations in either KRAS exons 3 or 4 or NRAS exons 2 or 3 (Figure 1 ).

\section{Association of RAS and BRAF mutations with clinicopathological features}

We analyzed the correlation between $R A S$ or BRAF genotypes and the clinicopathological features of mCRC. Primary rectal tumor tended to be more frequently observed in $K R A S$ exon 2 and other $R A S$ mutant tumors than in RAS wild-type tumors ( 60.0 vs. 61.9 vs. $48.3 \%, P=0.08$ ) (Table 1), although this was not statistically significant. $B R A F$ mutant tumors were more likely to develop in the right colon ( 57.1 vs. $18.0 \%, P=0.001$ ), and to have poorly differentiated or mucinous adenocarcinoma (42.9 vs. $7.2 \%$, $P=0.001$ ), and peritoneal metastasis (50.0 vs. $18.0 \%, P=$ 0.009 ) in comparison with BRAF wild-type tumors (Table 3).

\section{Efficacy of anti-EGFR therapies according to gene status Patient characteristics}

Between January 2013 and June 2014, 66 patients who met the inclusion criteria were treated with second- and third-line regimens containing anti-EGFR agents. Fifty-six patients had tumors with no mutations (all wild-type tumors) and 10 had tumors with mutation in either KRAS codons 61 or 146, NRAS, BRAF, or PIK3CA (any of the mutations). Among the 10 patients with any of the mutations, three had KRAS codon 146 mutations, two had $N R A S$ mutations, two had BRAF mutations, two had PIK3CA mutations (1 in exon 9 and 1 in exon 20), and one had BRAF and PIK3CA exon 9 mutations (Table 4).

Patients with any of the mutations were more likely to have worse PS and to be treated with anti-EGFR monotherapy than combination in comparison with all wild-type tumors. No other significant difference was seen between the two groups (Table 4).

\section{Response to treatment}

Among patients with all wild-type tumors $(n=56)$, complete response, partial response, stable disease and progressive disease were observed in 0 (0\%), 15 (26.8\%), $29(51.8 \%)$ and $12(21.4 \%)$ patients, respectively. In contrast, among patients with any of the mutations $(n=10)$, complete response, partial response, stable disease and disease progression were observed in $0(0 \%), 0(0 \%), 5$ $(50.0 \%)$ and 5 (50.0\%) patients, respectively. Thus, RR of patients with all wild-type tumors $(n=56)$ and those with any of the mutations $(n=10)$ were $26.8 \%$ and $0 \%$ $(P=0.101)$, respectively. Although DCR did not differ significantly between the two groups (78.6 vs. $50.0 \%, P=$ $0.109)$, DCR with complete or partial response and stable disease after $>3$ months was significantly better in patients with all wild-type tumors than in those with any of the mutations (76.8 vs. $10 \%, P=0.019$ ).

Among the 10 patients with mutations, three were treated with second-line anti-EGFR-containing regimens and seven were treated with third-line regimens. All three patients treated with second-line anti-EGFR therapy were

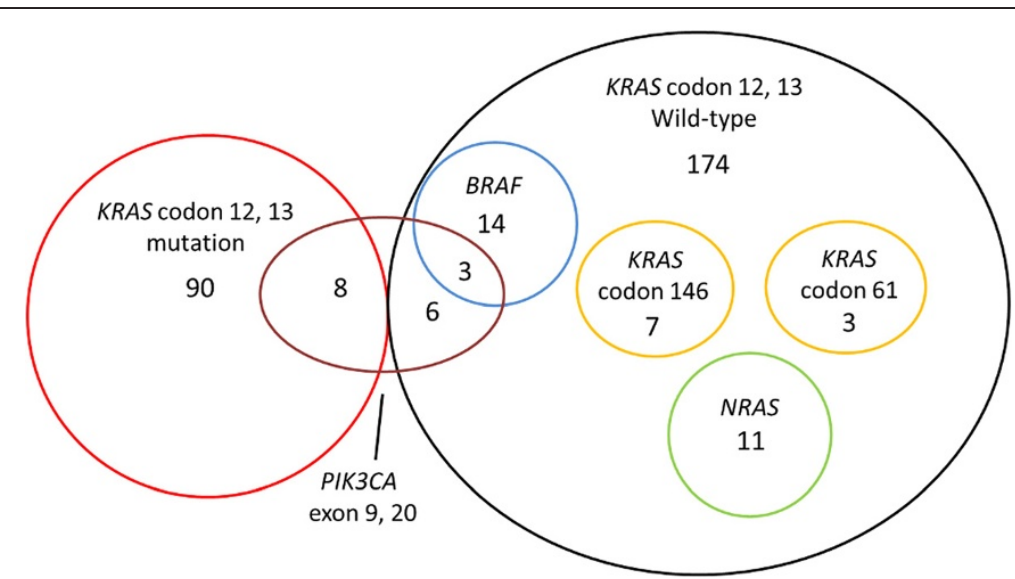

Figure 1 Associations between KRAS, NRAS, BRAF and PIK3CA mutations. All mutations in KRAS codons 12 and 13, KRAS codons 61 and 146, BRAF and NRAS were mutually exclusive. Mutations in PIK3CA exons 9 and 20 overlapped with those in KRAS codons 12 and 13 and BRAF. 
Table 3 Clinicopathological features according to BRAF mutations

\begin{tabular}{|c|c|c|c|c|}
\hline \multicolumn{2}{|l|}{ Characteristics } & $\begin{array}{l}\text { BRAF WT } \\
(n=250, \%)\end{array}$ & $\begin{array}{l}\text { BRAF MT } \\
(n=14, \%)\end{array}$ & $P$ value \\
\hline & & & & \\
\hline Age & Median (range) & $64(32-86)$ & 64 (46-75) & $0.53^{*}$ \\
\hline Gender & Male & $160(64.0)$ & $6(42.9)$ & $0.15^{* *}$ \\
\hline \multirow[t]{3}{*}{ Primary lesion } & Right-sided colon & $45(18.0)$ & $8(57.1)$ & $0.001^{* *}$ \\
\hline & Left-sided colon & $67(26.8)$ & $3(21.4)$ & \\
\hline & Rectum & $138(55.2)$ & $3(21.4)$ & \\
\hline \multirow[t]{2}{*}{ Histology } & Well, mod & $232(92.8)$ & $8(57.1)$ & $0.001^{* *}$ \\
\hline & Por, muc & $18(7.2)$ & $6(42.9)$ & \\
\hline \multirow[t]{4}{*}{ Site of metastasis } & Liver & $131(52.4)$ & $6(42.9)$ & $0.58^{* *}$ \\
\hline & Lung & 97 (38.8) & $3(21.4)$ & $0.26^{* *}$ \\
\hline & Lymph node & $143(57.2)$ & $7(50.0)$ & $0.59^{* *}$ \\
\hline & Peritoneum & 45 (18.0) & $7(50.0)$ & 0.009 \\
\hline
\end{tabular}

*Mann-Whitney $U$ test; ${ }^{* *} X^{2}$ or Fisher exact test.

irinotecan-naïve and had stable disease. Among these, one was treated with irinotecan plus panitumumab and showed stable disease after $>3$ months. In contrast, all seven patients treated with third-line anti-EGFR therapy were irinotecan refractory and only two had stable disease at $<3$ months.

\section{Survival analysis}

The median PFS of patients with any of the mutations ( $n=10 ; 2.2$ months; 95\% CI, 1.9-2.5 months) was significantly shorter than that of patients with all wild-type tumors ( $n=56$; 5.8 months; 95\% CI, 4.8-6.7 months), as verified in both univariate (HR 3.38; 95\% CI, 1.656.93; $P=0.001)$ and multivariate analyses (HR 2.77; 95\% CI, 1.16-6.61; $P=0.021$ ) (Figure 2A, Table 5).

The median OS of patients with any of the mutations ( $n=10$; 5.2 months; $95 \% \mathrm{CI}, 3.8-6.6$ months) was significantly shorter than that of patients with all wild-type tumors ( $n=56$; 17.7 months; $95 \%$ CI, $1.1-34.3$ months), as verified in both univariate (HR 4.94; 95\% CI, 2.12-11.5;

Table 4 Characteristics of patients who received anti-EGFR therapy

\begin{tabular}{|c|c|c|c|c|}
\hline \multicolumn{2}{|l|}{ Characteristics } & $\begin{array}{l}\text { All WT } \\
(n=56, \%)\end{array}$ & $\begin{array}{l}\text { Any } \mathrm{MT}^{\mathrm{b}} \\
(n=10, \%)\end{array}$ & $P$ value \\
\hline & & & & \\
\hline & TVlealan (range) & $64(34-19)$ & $64(51-14)$ & $0.629^{\pi}$ \\
\hline \multirow[t]{2}{*}{ ECOG PS } & 0 & $38(67.9)$ & $3(30.0)$ & $0.034^{* *}$ \\
\hline & $1-2$ & $18(32.1)$ & $7(70.0)$ & \\
\hline \multirow[t]{2}{*}{ Primary lesion } & Colon & $35(62.5)$ & $5(50.0)$ & $0.498^{* *}$ \\
\hline & Rectum & $21(37.5)$ & $5(50.0)$ & \\
\hline \multirow[t]{2}{*}{ Histology } & Well, mod & $53(94.6)$ & $8(80.0)$ & $0.162^{* *}$ \\
\hline & Por, muc & $3(5.4)$ & $2(20.0)$ & \\
\hline \multirow[t]{2}{*}{ Number of metastasis } & 1 & $14(25.0)$ & $4(40.0)$ & $0.442^{* *}$ \\
\hline & $>2$ & $42(75.0)$ & $6(60.0)$ & \\
\hline \multirow[t]{2}{*}{ Treatment line of anti-EGFR mab } & 2nd line & $27(48.2)$ & $3(30.0)$ & $0.327^{* *}$ \\
\hline & 3rd line & $29(51.8)$ & $7(70.0)$ & \\
\hline \multirow[t]{2}{*}{ Treatment } & Combination therapy & 44 (78.6) & $3(30.0)$ & $0.004^{* *}$ \\
\hline & Monotherapy & $12(21.4)$ & $7(70.0)$ & \\
\hline \multirow[t]{4}{*}{ Gene mutation } & KRAS codon 61, 146 & - & 3 & \\
\hline & NRAS codon $12,13,61$ & - & 2 & \\
\hline & PIK3CA exon 9, 20 & - & 3 & \\
\hline & BRAF codon 600 & - & 3 & \\
\hline
\end{tabular}




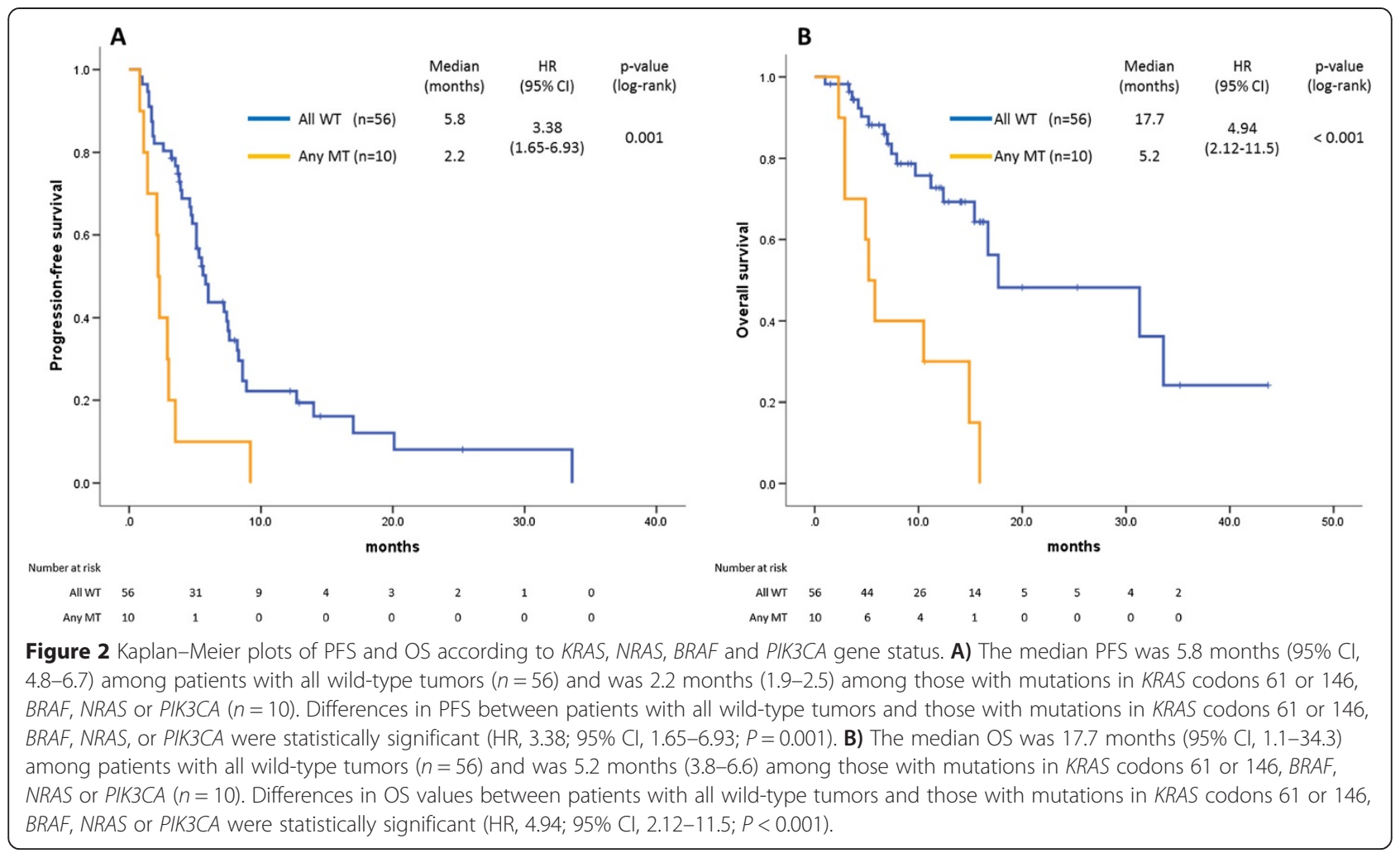

$P<0.001$ ) and multivariate analyses ( $\mathrm{HR} 3.38 ; 95 \% \mathrm{CI}$, 1.19-9.58; $P=0.022$ ) (Figure 2B, Table 5).

\section{Discussion}

We elucidated the prevalence of KRAS, NRAS, BRAF and PIK3CA mutations in Japanese $\mathrm{MCRC}$ patients, and clarified the relationship between gene status and clinicopathological features, including the efficacy of antiEGFR therapy. To date, clinical evidence about these mutations in mCRC has been based on clinical studies in western countries. The present study is believed to be the first to provide information on frequency and type of KRAS, NRAS, BRAF and PIK3CA mutations in Japanese patients with mCRC. In addition, the clinical feasibility of the present novel multiplex kit was demonstrated.

In our patient cohort, the frequency of patients with KRAS exon 2 (34.1\%) mutant tumors was similar to that in previous studies [2-4]. A total of $12.1 \%$ of patients without $K R A S$ exon 2 mutations had other $R A S$ mutations, which was lower than that in recent studies from western countries, which showed $15-26 \%$ of these mutations [12-18]. Another previous study from Japan showed that other $R A S$ mutations were detected in seven (12.7\%) of 55 samples without $K R A S$ exon 2 mutations with $3-13 \%$ sensitivity [26], which was similar to our result. Several possible explanations for the relatively lower frequency of other $R A S$ mutations in our study compared with western studies might be considered. First, there were some differences in detectable RAS mutations by multiplex kit between our study and western studies. In our study, we did not analyze KRAS codons 59 and 117 and NRAS codons 59, 117 and 146 , while these codons were analyzed in most western studies. Although the frequencies of these mutations are considered to be low, it might be one of the causes of the lower frequency in our patient cohort. Second, the sensitivity of RAS mutation analysis may vary among studies. In the present study, all mutations were detectable with 5$10 \%$ sensitivity. In contrast, Surveyor Scan Kits, BEAMing technology and pyrosequencing were used in pivotal studies, and RAS mutations were detected with 1-10\% sensitivity [12-18]. A recent multicenter study in Japan, including our institution, showed that other RAS mutations were detected in $15 \%$ of patients with KRAS exon 2 wild type, using a newer multiplex kit (MEBGEN RASKET Kit) [27]. This method detected 48 RAS mutations in exon 2 (codons 12 and 13), exon 3 (codons 59 and 61) and exon 4 (codons 117 and 146), with $1-5 \%$ sensitivity in a single reaction using 50-100-ng DNA from FFPE tissue without manual dissection. Given these methodological differences, further studies are required to confirm differences in the prevalence of other RAS mutations between Asian and western populations. In this study, we detected $B R A F$ mutations in $5.4 \%$ of patients. The prevalence of $B R A F$ mutation might be dependent on the patient population studied. mCRC patients with $B R A F$ mutant tumors have a poor prognosis, so the prevalence of $B R A F$ mutant populations may decline in 
Table 5 Univariate and multivariate analyses of PFS and OS

\begin{tabular}{|c|c|c|c|c|c|c|c|c|c|c|c|c|}
\hline \multirow[t]{2}{*}{ Variables } & & \multirow[t]{2}{*}{ No } & \multirow{2}{*}{$\begin{array}{l}\text { mPFS } \\
\text { (months) }\end{array}$} & \multicolumn{2}{|c|}{ Univariate analysis } & \multicolumn{2}{|c|}{ Multivariate analysis } & \multirow{2}{*}{$\begin{array}{l}\text { mOS } \\
\text { (months) }\end{array}$} & \multicolumn{2}{|c|}{ Univariate analysis } & \multicolumn{2}{|c|}{ Multivariate analysis } \\
\hline & & & & HR $(95 \% \mathrm{Cl})$ & $P$ value & HR $(95 \% \mathrm{Cl})$ & $P$ value & & $\mathrm{HR}(95 \% \mathrm{Cl})$ & $P$ value & HR $(95 \% \mathrm{Cl})$ & $P$ value \\
\hline \multirow[t]{2}{*}{ Mutation status } & All WT & 56 & 5.8 & reference & - & reference & - & 17.7 & reference & - & reference & - \\
\hline & Any MT & 10 & 2.2 & $3.38(1.65-6.93)$ & 0.001 & $2.77(1.16-6.61)$ & 0.021 & 5.2 & $4.94(2.12-11.5)$ & $<0.001$ & 3.38 (1.19-9.58) & 0.022 \\
\hline \multirow[t]{2}{*}{ ECOG PS } & 0 & 41 & 6.0 & reference & - & reference & - & 31.3 & reference & - & reference & - \\
\hline & $1-2$ & 25 & 3.5 & $2.82(1.53-5.20)$ & 0.001 & $1.80(0.89-3.64)$ & 0.101 & 9.7 & $3.41(1.52-7.69)$ & 0.003 & $1.62(0.59-4.42)$ & 0.346 \\
\hline \multirow[t]{2}{*}{ Number of metastasis } & 1 & 18 & 6.0 & reference & - & reference & - & 15.4 & reference & - & reference & - \\
\hline & $>2$ & 48 & 5.1 & $1.57(0.82-3.00)$ & 0.17 & $1.51(0.73-3.11)$ & 0.268 & 16.7 & $1.70(0.68-4.29)$ & 0.257 & $1.60(0.59-4.30)$ & 0.353 \\
\hline \multirow[t]{2}{*}{ Treatment line of anti-EGFR mab } & 2nd & 30 & 7.6 & reference & - & reference & - & 17.7 & reference & - & reference & - \\
\hline & $3 r d$ & 36 & 4.0 & $1.85(1.06-3.25)$ & 0.032 & $1.52(0.83-2.79)$ & 0.174 & 15.9 & $1.43(0.66-3.10)$ & 0.367 & $0.97(0.41-2.29)$ & 0.940 \\
\hline \multirow[t]{2}{*}{ Combination therapy } & Yes & 47 & 7.4 & reference & - & reference & - & 31.3 & reference & - & reference & - \\
\hline & No & 19 & 2.6 & $4.82(2.49-9.35)$ & $<0.001$ & $2.73(1.28-5.83)$ & 0.009 & 10.5 & 3.31 (1.48-7.41) & 0.004 & $2.03(0.83-4.96)$ & 0.121 \\
\hline
\end{tabular}

mOS, median overall survival; mPFS, median progression-free survival. 
pretreated patients compared with chemonaïve patients. The prevalence of $B R A F$ mutations in our patient cohort was similar to that of previous studies of pretreated patients with mCRC [11,12,19-24].

We also investigated the clinicopathological features of mCRC patients with respect to RAS and BRAF mutations. Primary rectal tumor tends to be more frequently observed in KRAS exon 2 and other RAS mutant tumors rather than $R A S$ wild-type tumors, although this was not statistically significant. Previous studies showed that KRAS exon 2 mutation was significantly higher in the right colon [28,29], in disagreement with our analysis. No significant differences in other clinicopathological features such as age, sex, primary lesion, histology, and site of metastasis were observed between KRAS exon 2 and other RAS mutant tumors, which is similar to previous studies [30]. Regardless of these clinicopathological features, it is reported that other gene expression profiles based on The Cancer Genome Atlas appear to be similar in patients with KRAS and NRAS mutant mCRC, suggesting that treatment selection based on molecular profile is important [30]. In accordance with previous reports [23,24], BRAF mutant tumors are more likely to develop in the right colon, and to have poorly differentiated or mucinous adenocarcinoma, and peritoneal metastasis in comparison with BRAF wild-type tumors.

In agreement with previous studies [19,25], mutations in KRAS exons 3 or 4, NRAS, BRAF or PIK3CA were not associated with clinical benefits from anti-EGFR therapy in the present cohort. On the basis of recent prospective and retrospective randomized trials of anti-EGFR therapy [12-18], the National Comprehensive Cancer Network (NCCN) recommends anti-EGFR therapy for mCRC patients without other RAS mutant tumors or KRAS exon 2 mutant tumors [31]. The Japanese Society of Medical Oncology (JSMO) also recommends testing for all RAS mutations in patients with $\mathrm{mCRC}$ before anti-EGFR therapy. In contrast, whether $B R A F$ and PIK3CA mutations are predictive of the efficacy of anti-EGFR therapy remains controversial [19-22]. Previous trials suggest that intensive combination chemotherapy with FOLFOXIRI (5-FU, Lleucovorin, irinotecan, and oxaliplatin) and bevacizumab might be especially effective for $B R A F$ mutant mCRC [32]. Recently, the combination of $B R A F$ inhibitors and antiEGFR monoclonal antibodies, with or without PI3K inhibitors or MEK inhibitors, has shown promising results in phase I trials in patients with $B R A F$ mutant CRC [33,34]. Patients with $B R A F$ mutant CRC are often refractory to systematic chemotherapy and have poor prognosis, therefore, screening for $B R A F$ mutations is important during recruitment of patients for these clinical trials. Accordingly, we conducted a multi-institutional screening (GI-SCREEN) study using the present multiplex kit to elucidate the nationwide prevalence of these targetable mutations.
There were several methodological limitations to the present study. First, not all of the patients in our study period were evaluated for their RAS gene status. Thus, the analysis may have been subject to some selection bias. Second, the small sample size and single-center population were other major limitations. Owing to the overall small number of patients with KRAS exon 3 or 4, NRAS, BRAF or PIK3CA mutations, we could not evaluate the impact of each gene mutation on the efficacy of anti-EGFR therapy. In addition, our analyses were explorative and hypothesis generating. This issue should be analyzed in a larger cohort.

\section{Conclusions}

Other RAS and BRAF mutations have been observed in KRAS exon 2 wild-type tumors, which were associated with some clinicopathological features and resistance to anti-EGFR therapy in our patient cohort. Importantly, because there are a certain number of $\mathrm{mCRC}$ patients with molecular alteration other than KRAS exon 2, further refinement of tumor-specific genetic markers is needed to improve the efficacy of anti-EGFR therapy.

\section{Additional file}

Additional file 1: The genotypes of all samples using HGVS nomenclature.

\section{Abbreviations}

Cl: Confidence interval; CT: Computed tomography; DCR: Disease control rate; EGFR: Epidermal growth factor receptor; FFPE: Formalin-fixed, paraffinembedded; mCRC: Metastatic colorectal cancer; OS: Overall survival;

PCR: Polymerase chain reaction; PFS: Progression-free survival; RR: Response rate.

\section{Competing interests}

The authors declare that they have no competing interests.

\section{Authors' contributions}

KS and TY (Yoshino) conceived the study design. AK carried out the majority of molecular genetic studies and analyses of the clinical data. KS, SF, YK, HB, WO, TK, NF, TD, and TY (Yoshino) provided clinical data and helped collect tumor tissues. TY (Yamanaka) statistically analyzed the clinical data. AO coordinated the study and helped to draft the manuscript. All authors have read and approved the final manuscript.

\section{Acknowledgements}

This study was supported by a research funding from National Cancer Center Hospital East.

\section{Author details}

${ }^{1}$ Department of Gastroenterology and Gastrointestinal Oncology, National Cancer Center Hospital East, 6-5-1 Kashiwanoha, Kashiwa, Chiba 277-8577, Japan. ${ }^{2}$ Exploratory Oncology Research \& Clinical Trial Center, National Cancer Center, Chiba, Japan. ${ }^{3}$ Department of Biostatistics, Yokohama City University, Kanagawa, Japan.

Received: 29 September 2014 Accepted: 26 March 2015

Published online: 11 April 2015

\section{References}

1. International Agency for Research on Cancer. GLOBOCAN 2012 http://www-dep.iarc.fr/CancerMondial.htm. Accessed on 29/08/2014. 
2. Andreyev HJ, Norman AR, Cunningham D, Oates JR, Clarke PA. Kirsten ras mutations in patients with colorectal cancer: the multicentre "RASCAL" study. J Natl Cancer Inst. 1998;90:675-84.

3. Forbes S, Clements J, Dawson E, Bamford S, Webb T, Dogan A, et al. COSMIC 2005. Br J Cancer. 2006;94:318-22.

4. Scheffzek K, Ahmadian MR, Kabsch W, Wiesmüller L, Lautwein A, Schmitz F, et al. The Ras-RasGAP complex: structural basis for GTPase activation and its loss in oncogenic Ras mutants. Science. 1997;277:333-8.

5. Trahey M, McCormick F. A cytoplasmic protein stimulates normal NRAS p21 GTPase, but does not affect oncogenic mutants. Science. 1987;238:542-5.

6. Amado RG, Wolf M, Peeters M, Van Cutsem E, Siena S, Freeman DJ, et al. Wild-type KRAS is required for panitumumab efficacy in patients with metastatic colorectal cancer. J Clin Oncol. 2008;26:1626-34.

7. Karapetis CS, Khambata-Ford S, Jonker DJ, O'Callaghan CJ, Tu D, Tebbutt NC, et al. K-ras mutations and benefit from cetuximab in advanced colorecta cancer. N Engl J Med. 2008;359:1757-65.

8. Van Cutsem E, Kohne CH, Hitre E, Zaluski J, Chang Chien CR, Makhson A et al. Cetuximab and chemotherapy as initial treatment for metastatic colorectal cancer. N Engl J Med. 2009;360:1408-17.

9. Douillard JY, Siena S, Cassidy J, Tabernero J, Burkes R, Barugel M, et al. Randomized, phase III trial of panitumumab with infusional fluorouracil, leucovorin, and oxaliplatin (FOLFOX4) versus FOLFOX4 alone as first-line treatment in patients with previously untreated metastatic colorectal cancer: the PRIME study. J Clin Oncol. 2010;28:4697-705.

10. Peeters M, Price TJ, Cervantes A, Sobrero AF, Ducreux M, Hotko Y, et al. Randomized phase III study of panitumumab with fluorouracil, leucovorin, and irinotecan (FOLFIRI) compared with FOLFIRI alone as second-line treatment in patients with metastatic colorectal cancer. J Clin Oncol. 2010;28:4706-13.

11. Vaughn CP, Zobell SD, Furtado LV, Baker CL, Samowitz WS. Frequency of KRAS, BRAF, and NRAS mutations in colorectal cancer. Genes Chromosomes Cancer. 2011;50:307-12.

12. Douillard JY, Oliner KS, Siena S, Tabernero J, Burkes R, Barugel M, et al. Panitumumab-FOLFOX4 treatment and RAS mutations in colorectal cancer. N Engl J Med. 2013;369:1023-34.

13. Peeters M, Oliner KS, Price TJ, Cervantes A, Sobrero AF, Ducreux M, et al. Analysis of KRAS/NRAS mutations in phase 3 study 20050181 of panitumumab (pmab) plus FOLFIRI versus FOLFIRI for second-line treatment (tx) of metastatic colorectal cancer (mCRC). J Clin Oncol. 2014;32 suppl 3:bstr LBA387.

14. Patterson SD, Peeters M, Siena S, Van Cutsem E, Humblet $Y$, Van Laethem J-L, et al. Comprehensive analysis of KRAS and NRAS mutations as predictive biomarkers for single agent panitumumab (pmab) response in a randomized, phase 3 metastatic colorectal cancer (mCRC) study (20020408). J Clin Oncol. 2013;31(suppl):abstr 3617.

15. Tejpar S, Lenz HJ, Köhne CH, Heinemann V, Ciardiello F, Esser R, et al. Effect of KRAS and NRAS mutations on treatment outcomes in patients with metastatic colorectal cancer (mCRC) treated first-line with cetuximab plus FOLFOX4: New results from the OPUS study. J Clin Oncol. 2014;32 suppl 3:abstr LBA444.

16. Heinemann V, von Weikersthal LF, Decker T, Kiani A, Vehling-Kaiser $U$, Al-Batran SE, et al. FOLFIRI plus cetuximab versus FOLFIRI plus bevacizumab as first-line treatment for patients with metastatic colorectal cancer (FIRE-3): a randomised, open-label, phase 3 trial. Lancet Oncol. 2014;15:1065-75.

17. Schwartzberg LS, Rivera F, Karthaus M, Fasola G, Canon JL, Hecht JR, et al. PEAK: a randomized, multicentre phase II study of panitumumab plus modified fluorouracil, leucovorin, and oxaliplatin (mFOLFOX6) or bevacizumab plus mFOLFOX6 in patients with previously untreated, unresectable, wild-type KRAS exon 2 metastatic colorectal cancer. J Clin Oncol. 2014;32:2240-7.

18. Ciardiello F, Lenz H-F, Kohne C-H, Heinemann V, Tejpar S, Melezinek I, et al. Treatment outcome according to tumour RAS mutation status in CRYSTAL study patients with metastatic colorectal cancer (mCRC) randomized to FOLFIRI with/without cetuximab. J Clin Oncol. 2014;32:5s. suppl; abstr 3506.

19. De Roock W, Claes B, Bernasconi D, De Schutter J, Biesmans B, Fountzilas G, et al. Effects of KRAS, BRAF, NRAS, and PIK3CA mutations on the efficacy of cetuximab plus chemotherapy in chemotherapy-refractory metastatic colorectal cancer: a retrospective consortium analysis. Lancet Oncol. 2010;11:753-62.

20. Van Cutsem E, Köhne CH, Láng I, Folprecht G, Nowacki MP, Cascinu S, et al. Cetuximab plus irinotecan, fluorouracil, and leucovorin as first-line treatment for metastatic colorectal cancer: updated analysis of overall survival according to tumour KRAS and BRAF mutation status. J Clin Oncol. 2011;29:2011-9.
21. Sartore-Bianchi A, Martini M, Molinari F, Veronese S, Nichelatti M, Artale S, et al. PIK3CA mutations in colorectal cancer are associated with clinical resistance to EGFR-targeted monoclonal antibodies. Cancer Res. 2009;69:1851-7.

22. Karapetis CS, Jonker D, Daneshmand M, Hanson JE, O'Callaghan CJ, Marginean C, et al. PIK3CA, BRAF, and PTEN status and benefit from cetuximab in the treatment of advanced colorectal cancer-results from NCIC CTG/AGITG CO.17. Clin Cancer Res. 2014;20:744-53.

23. Yokota T, Ura T, Shibata N, Takahari D, Shitara K, Nomura M, et al. BRAF mutation is a powerful prognostic factor in advanced and recurrent colorectal cancer. Br J Cancer. 2011;104:856-62.

24. Zlobec I, Bihl MP, Schwarb H, Terracciano L, Lugli A. Clinicopathological and protein characterization of BRAF- and K-RAS-mutated colorectal cancer and implications for prognosis. Int J Cancer. 2010;127:367-80.

25. Bando H, Yoshino T, Shinozaki E, Nishina T, Yamazaki K, Yamaguchi K, et al. Simultaneous identification of 36 mutations in KRAS codons 61 and 146, BRAF, NRAS, and PIK3CA in a single reaction by multiplex assay kit. BMC Cancer. 2013;13:405.

26. Ishige T, Itoga S, Sato K, Kitamura K, Nishimura M, Sawai S, et al. Highthroughput screening of extended RAS mutations based on high-resolution melting analysis for prediction of anti-EGFR treatment efficacy in colorectal carcinoma. Clin Biochem. 2014:47:340-3.

27. Kudo T, Satoh T, Muro K, Taniguchi H, Nishina T, Kajiwara T, et al. Clinical validation of a novel multiplex kit for all RAS mutations in colorectal cancer: Results of RASKET (RAS KEY Testing) prospective multicentre study. Ann Oncol. 2014;25 Suppl 2:ii14-104.

28. Barault L, Veyrie N, Jooste V, Lecorre D, Chapusot C, Ferraz JM, et al. Mutations in the RAS-MAPK, PI(3)K (phosphatidylinositol-3-OH kinase) signaling network correlate with poor survival in a population-based series of colon cancers. Int J Cancer. 2008;122:2255-9.

29. Watanabe $T$, Yoshino $T$, Uetake $H$, Yamazaki $K$, Ishiguro M, Kurokawa T, et al. KRAS mutational status in Japanese patients with colorectal cancer: results from a nationwide, multicentre, cross-sectional study. Jpn J Clin Oncol. 2013;43:706-12.

30. Morris VK, San Lucas FA, Overman MJ, Eng C, Morelli MP, Jiang ZQ, et al. Clinicopathologic characteristics and gene expression analyses of non-KRAS 12/13, RAS-mutated metastatic colorectal cancer. Ann Oncol. 2014;25:2008-14.

31. NCCN Clinical Practice Guidelines in Oncology_Colon Cancer, Rectal Cancer Version 1.2015. http://www.nccn.org/professionals/physician_gls/pdf/colon. pdf. Accessed on 29/08/2014.

32. Loupakis F, Cremolini C, Salvatore L, Masi G, Sensi E, Schirripa M, et al. FOLFOXIRI plus bevacizumab as first-line treatment in BRAF mutant metastatic colorectal cancer. Eur J Cancer. 2014;50:57-63.

33. Van Geel R, Elez E, Bendell JC, Faris JE, Lolkema MPJK, Eskens F, et al. Phase study of the selective BRAFV600 inhibitor encorafenib (LGX818) combined with cetuximab and with or without the a-specific PI3K inhibitor BYL719 in patients with advanced BRAF-mutant colorectal cancer. J Clin Oncol. 2014;32:5s. suppl; abstr 3514.

34. Bendell JC, Atreya CE, André T, Tabernero J, Gordon MS, Bernards R, et al. Efficacy and tolerability in an open-label phase $1 /$ II study of MEK inhibitor trametinib (T), BRAF inhibitor dabrafenib (D), and anti-EGFR antibody panitumumab (P) in combination in patients (pts) with BRAF V600E mutated colorectal cancer (CRC). J Clin Oncol. 2014;32:5s. suppl; abstr 3515.

\section{Submit your next manuscript to BioMed Central and take full advantage of:}

- Convenient online submission

- Thorough peer review

- No space constraints or color figure charges

- Immediate publication on acceptance

- Inclusion in PubMed, CAS, Scopus and Google Scholar

- Research which is freely available for redistribution 